\title{
Self adjustable security technique based on remote reference passing for digital multimedia services
}

\author{
Darshana Hooda $\cdot$ Parvinder Singh
}

Received: 25 January 2013/Accepted: 3 May 2013/Published online: 31 May 2013

(C) CSI Publications 2013

\begin{abstract}
In this paper, we have proposed parameterized security technique based on remote reference passing (RRP). RRP intrinsically works on the principle of transmitting the information or data in the form of reference at receiver side. RRP is most suitable technique to address security needs of video services while accessed/played through constrained devices because of its low power design and negligible delay during decryption process, one of stringent requirement of video transmission. Proposed technique has potential to offer varying level of perceptual encryption which makes it suitable to support different multimedia service with different security needs. This technique offer confidentiality and authentication for secure communication and further, same is also suitable for entertainment video services to facilitate pay TV, video on demand or pay per view. This technique encrypts the raw video in RGB 24 bit color space which makes its integration possible with different video codec.
\end{abstract}

Keywords Video encryption - Remote reference passing . Multimedia services

\section{Introduction}

Recent advances in multimedia compression and communication technologies and abundant availability of low cost limited capabilities display devices have led to a phenomenal growth of digital multimedia services and applications like

\author{
D. Hooda $(\varangle) \cdot$ P. Singh \\ Deenbandhu Chhotu Ram University of Science \\ and Technology, Murthal, Sonepat, Haryana, India \\ e-mail: dhooda.ucc@dcrustm.org \\ P. Singh \\ e-mail: drparvindersingh.cse@dcrustm.org
}

video chat, video conferencing, video broadcasting, video on demand, online movie transmission, virtual education, medical imaging systems and video transmission on mobiles using $3 \mathrm{G}$ services. Multimedia services can be broadly classified into entertainment service and communication services as per the security needs. Both categories have different set of security requirements. Therefore there is strong need to design and develop new techniques and technologies not only to protect valuable multimedia assets from unauthorized access but to address new challenges like limited computing capabilities of display devices and different security needs of multimedia services.

Crypto Algorithms are the core of conventional security system however watermarking and finger printing techniques are evolving rapidly to address security needs of multimedia applications which falls under entertainment category. Conventional Crypto Algorithms are typically too slow for real time applications. Situation is even worse when they are used in the environments, where receiving end devices may have very limited resources or low computational power.

In many cases the level of protection of information is much higher than it actually requires to meeting potential threat. As the computational needs increases with the level of security imposed, this strongly affects the performance of application. Excessive protection coupled with low computational processing capability of end access device introduces undesirable delay during the processing of received data. This delay is highly undesirable for real time application. Therefore it is critical to apply optimal level of security taking under consideration of limited resources of receiving end device.

Conventional cryptographic algorithms, which generally aim at encrypting text data, however, are not well suited for video encryption. This is due to the fact that conventional crypto algorithms cannot process the large amount of video 
data in real time. From above discussion it is concluded that there is need to design parameterized encryption algorithm to control the perceptual encryption to address different security aspects of multimedia services. Entertainment applications have loose security needs while personalized video services require high degree of security. The value of video data in entertainment/virtual education applications is associated with video quality and timeliness. In entertainment industry high quality video is priced and requires an authorized access, while low quality versions or little disguised videos may free, to stimulate the user to purchase high quality version [1]. Personalized video applications are sensitive applications and usually have strict security requirements equal to those demanded for text encryption. The encryption algorithms for personalized video services have to withstand not only classical cryptanalytic attacks but also the perceptual attack [2, 3] in order to ensure that no visible information related to the sensitive communication is disclosed.

This paper targets the perceptual encryption of video stream and presents designing of parameterized encryption based on the cryptographic model which works on concept of remote reference passing (RRP) as shown in Fig. 1. This model is best suitable to provide security to multimedia applications, where level of security is extended as per the receiving device resources and multimedia service requirement.

This paper is organized as follows: in Sect. 2 RRP mechanism is discussed in detail. Next section covers designing of proposed technique. Experimental study is presented in Sect. 4 to show encryption strength and encryption-decryption time. The last section presents the conclusion.

\section{Background: remote reference passing framework}

Remote reference passing framework is based on client server architecture. Input source of video data is a high processing capability server while accessing devices termed as clients are having very limited computational capabilities. Under said framework, to achieve secure communication, all the processing overhead is kept on the server while minimum overhead on the client machine taking into account its limited processing capability.

In video transmission, delay may be accumulated due to transmission delay, compression/decompression delay and if security is needed then it adds encryption/decryption delay, further. Therefore objective of all the research dealing with video transmission significantly takes care about delay introduced by the proposed method either it is associated with compression or security. All these techniques should converge to minimize delay during video data transmission so that video can be enjoyed at full. In literature of said framework it is critically stated by the author that RRP framework introduces negligible delay while secure video is accessed/played by the constrained receiver, further it has potential to offer different level and kind of security for different digital video services.

Most exciting feature of this model is that it introduces negligible delay at receiving end for long duration video. Generally high level security introduces more overhead; but in this model low and high level of security, both incur same amount of overhead on receiver. However at server, all the remote reference creation takes place hence it introduces delay and this delay varies as per security scheme. Sender systems are high end machines so delay introduced by it, comparable to existing security techniques. Details of working of model is illustrated in Fig. 1 and further working of each step is described in detail:

\subsection{Buffer allocation (video sink module)}

Video sink prepares a buffer by placing data values in buffer $\mathrm{B}$ of size $\mathrm{N}$ using mathematical mapping between index and candidate video data values. Next receiver transmits $\mathrm{N}$ (size of buffer), Val $\left(0-2^{\mathrm{N}}\right)$ and $\mathrm{B}_{0}$ (random index of $\mathrm{B}$ ) these keys along the request to Video Source to transmit intended video stream:

Fig. 1 Working of Proposed Model

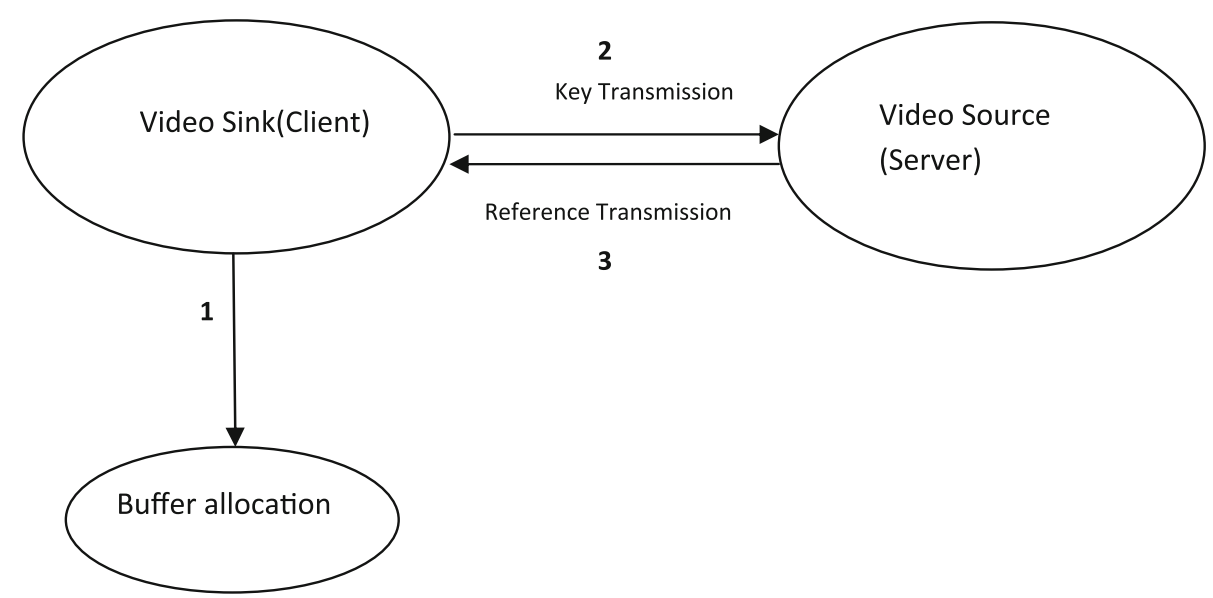


For buffer allocation many more scheme may be applied as per security need and availability of memory.

Buffer allocation is offline activity just prior to the actual transmission. Before each new demand new buffer allocation will takes place that adds more security or we can say per new request key changes. However actual implementation and use of buffer have many dimensions like during the decryption buffer may copied into an array or starting address of buffer may be transferred to processor register or complete buffer may be transferred to cache for fast access, hence references will be used accordingly. This is client specific activity prior to actual data transmission, which takes place offline therefore does not affect decryption time during real time video consumption. This offline time for buffer preparation is also negligible as the size of buffer remains in between 256 to 512 . Whenever size increases from 256 there is increase in file size.

\subsection{Key transmission}

After preparation of buffer at video sink, $\mathrm{B}_{0}, \mathrm{Val}_{1}, \mathrm{~N}$ are the keys obtained from step 1 . These keys are conveyed to video source as indicated in step 2 using any conventional method. One way key exchange from client to server is needed.

\subsection{Remote reference creation module (video source module)}

Video source creates remote references for the intended input video for video sink with the help of received keys from same through following module. Sever replaces the data value by the index of buffer (B) at client, where this value resides with the help of predetermined formula/algorithm.

\subsection{Video sink module(client module)}

Reference for video input is received by the video sink and original video stream is obtained by referring the address where actual data is available. Beauty of algorithm that client is not receiving the data but a pointer which indicates a location where referred data is available.

\section{Proposed technique}

Proposed self adjustable technique works on the principles of number theory such as congruence and residue classes [4].

\subsection{Residue class}

Integers $\mathrm{p}$ and $\mathrm{q}$ are said to be members of the same residue class $(\bmod \mathrm{m})$ when they have the same principle remainder $(\bmod \mathrm{n})$ corresponding to the $\mathrm{n}$ principle remainders $0,1, \ldots, n-1$. a and $b$ belong to same residue class $\bmod \mathrm{n}$ iff $\mathrm{a} \equiv \mathrm{b}(\bmod \mathrm{n})$.

\subsection{Complete residue system}

Any set of integers $\left\{a_{1}, a_{2}, \ldots, a_{n}\right\}$ representing all the residue classes $(\bmod n)$. The simplest complete residue system is $0,1, \ldots, n-1$ for $\bmod n$.

Proposed technique are based on following theorem from number theory.

\subsection{Theorem}

- Let $\mathrm{n}$ be a positive integer. let $\mathrm{a}$ and $\mathrm{b}$ be an integer and let $\mathrm{k} \equiv \operatorname{gcd}(\mathrm{a}, \mathrm{n})$. Then the congruence $\mathrm{ax} \equiv \mathrm{r}(\bmod \mathrm{n})$ has $\mathrm{k}$ solutions or no solutions according as $\mathrm{klr}$ or $\mathrm{k}+\mathrm{r}$.

From above theorem it is clear that if $(a, n)$ are coprime i.e. $\operatorname{gcd}(\mathrm{a}, \mathrm{n})=1$ then $\mathrm{ax} \equiv \mathrm{b}(\bmod \mathrm{n})$ has only one solution for all $b=\{0,1,2, \ldots, n-1\}$.

Aforesaid principle is used to correlate the buffer index and value both over $\mathrm{I}=\{1, \ldots, \mathrm{N}\}$, during the buffer creation by the client module.

\subsection{Residue classes of integers mod $n$}

The congruence relation $\mathrm{p} \equiv \mathrm{r}(\bmod \mathrm{n})$ on the set of integers I separate the integers into $n$ equivalence classes $[0]_{\mathrm{n}},[1]_{\mathrm{n}},[2]_{\mathrm{n}},[3]_{\mathrm{n}}, \ldots,[\mathrm{n}-1]_{\mathrm{n}}$ Called residue classes modulo n. Each equivalence class $[\mathrm{r}]_{\mathrm{n}}$ consists of all integers congruent to $r$ where $r$ is one of the integers $0,1,2, \ldots, \mathrm{n}-1$. These $\mathrm{n}$ integers $0,1,2, \ldots, \mathrm{n}-1$ are called the class representatives.

From above discussion we reach the conclusion that the congruence relation $\mathrm{pq} \equiv \mathrm{r}(\bmod \mathrm{n})$; where $\operatorname{gcd}(\mathrm{p}, \mathrm{n}) \equiv 1$ for all $\mathrm{n} \geq 256$ and for $\forall q=\{1,2,3, \ldots, \mathrm{n}\}$ generates unique single value of $r$ for each $q$ over integer set $\mathrm{I}=\{0,1,2, \ldots, n-1\}$. Further, This relation separates the $r$ set into $\mathrm{p}$ equivalence classes for $\mathrm{n} \bmod \mathrm{p}$, where each class contains I_n/p_l elements and $\left\{r, p+r, 2 p+r, I_{-} n / p \_l\right.$ $\mathrm{p}+\mathrm{r}\}$ for $\forall r$ with respect to $\mathrm{n} \bmod \mathrm{p}$ generates simple complete residue class $\bmod \mathrm{n}$.

The congruence relation $\mathrm{pq} \equiv \mathrm{r}(\bmod \mathrm{n})$ on the set of integers $r=\{0, \ldots, n-1\}$ separates the set $r$ into $p$ equivalence classes $[0]_{\mathrm{n} \bmod \mathrm{p}},[1]_{\mathrm{n} \bmod \mathrm{p}}, \ldots,[\mathrm{p}-1]_{\mathrm{n} \bmod \mathrm{p}}$ such that these $\mathrm{p}$ equivalence classes form a complete simple residue system for $\bmod n$ i.e. $\{0,1, \ldots, n-1\}$.

From above it is inferred, that for the congruence relation $\mathrm{pq} \equiv \mathrm{r}(\bmod \mathrm{n})$, where $\operatorname{gcd}(\mathrm{p}, \mathrm{n})=1$ for each $\mathrm{r}=\{0,1, \ldots, \mathrm{n}-1\}$ there exist unique $\mathrm{q}_{\mathrm{i}}=\left|\_\mathrm{n} / \mathrm{p}_{-}\right| \times$ $\bmod (r, p)+1+\frac{r-\bmod (r, p)}{p}$ iffn $\equiv 1(\bmod p)$ 


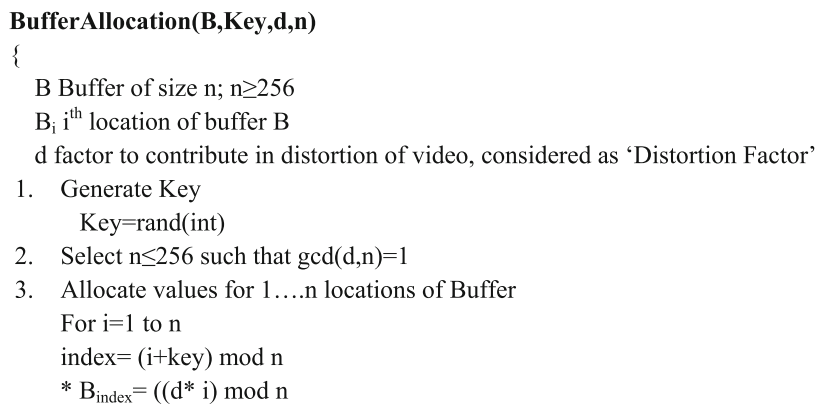

\section{Performance analysis and experiment results}

The two main characteristics that identify and differentiate one encryption algorithm from another are its ability to secure the protected data against attacks and its speed and efficiency in doing so. Complexities of encryption and decryption modules come under consideration, when ever efficiency of crypto algorithms is discussed. Our major consideration is time taken by the decryption module, keeping in the view limited computational capabilities of video sinks and real time processing need of video stream. However abundant availability of constrained devices for video access arise need for low power design encryption techniques. Proposed technique offer most effective security solution for various multimedia services in today's scenario.

For quantitative analysis of video encryption techniques, there is a need to define a set of performance parameter considered as video encryption metric. This metric helps the user to evaluate the performance of video encryption algorithm. We have presented complete performance evaluation of proposed technique based on following parameter, defines strength of proposed cryptographic technique.

\subsection{Computational efficiency}

It may be defined in terms of space complexity and time complexity of encryption algorithms. Space complexity of encryption is determined by memory requirement for code and data while time complexity measures the time requirement for encryption/decryption. Software and hardware implementation of cryptographic algorithms exits in plenty and both have different and sometimes contrary characteristics. For software implementation RAM and ROM requirements and required number of clock cycles, plays vital role in determining performance of algorithm. Situation becomes more critical in today's scenario when video data is displayed on constrained devices i. e. display devices with limited computing resources like memory, processing speed and power. It ignite the need of small size decryption algorithms code i.e. low space complexity as well as less time complexity to meet real time requirement of video applications while played on constrained devices.

Space for buffer adds additional need of memory as compared to the conventional crypto technique, but it is acceptable because of increased speed of the decryption process, one of stringent and utmost requirement of video encryption. In proposed technique code size foe decryption module is optimal in its size. However, proposed technique establishes tradeoff between security level and memory which is not a critical factor in today's world. In conventional crypto technique always tradeoff exists between security level and speed. In proposed technique speed of decryption is almost same for all level of security.

Simulation results are presented in next sub-Sect. 4.2 to show strength of proposed RRP based cryptography and conventional cryptography mechanism with respect to decryption process.

\subsection{Security strength}

Emerging video applications like $\mathrm{VoD}$, pay per view, videoconferencing, telemedicine etc. need different level/ type of security. VoD requires loose security needs with perceptual degradation only while video conferencing may require totally closed communication for all others out of communication group so high perceptual degradation is needed to totally disguise the video so adversaries can't perceive it. Proposed technique has potential to offer security for controlled access as well as for confidential communication. Encryption algorithms used in entertainment applications are considered secure and valuable if the two conditions firstly if the cost to break the algorithm is higher than the license fee for the video content and secondly if the time required to break the algorithm is longer than the time that the encrypted video. Key space is sufficiently large to resist brute-force attack and perceptual 
attacks in most cases are able to reconstruct just lowquality video whose perceptual value is not comparable to the original video. Therefore perceptual attacks do not pose a great threat to entertainment applications. For sensitive applications to prevent perceptual attacks proposed encryption technique encrypt the video in such a manner that no perceptual information is revealed to the adversaries. Further, In proposed technique client takes buffer of size $\mathrm{n}$ (contiguous memory locations) from its local memory space that is unknown to adversary hence adds one more layer to security.

Security offered by proposed method is also sufficient as it is proved that security is not hacked by breaking the encryption techniques but they are hacked due to flows in system itself. Therefore, now it is strongly recommend that a fast not very highly secure but reasonably good encryption technique also offer optimistic results and future.

Security of proposed method is

Let memory space of receiver is G, size of key is $\mathrm{K}=2^{\text {size of (unsigned integer) }}$, size of buffer $=\mathrm{N}$

Probability of breaking the system $=1 /(\mathrm{G} \times \mathrm{N} \times$ $2^{\text {size of (unsigned integer) }}$

From above discussion it is evident that problem of cryptanalysis for proposed method is NP hard. It is proven that there exists no polynomial time solution for NP hard problems. Therefore, it also gives strong recommendation to use proposed model to provide satisfactory level of security to different multimedia services, duly taking care of optimality principal in terms of delay and limited processing capability of end device. Further, to offer very high level of security more buffers may be used with different keys and Distortion Factor. There is tradeoff between memory requirement and security. However, no effect on decryption time i.e. neither delay increased nor computational complexity increased when security level is increased, one of the foremost goal of proposed technique.

Simulation results are presented in next sub-Sect. 4.2 to show perceptual distortion of proposed technique as result of variation in 'Distortion Factor' m.

\subsection{Compression efficiency}

Video data is generally very large so usually compressed to reduce storage space and to save bandwidth. Encryption algorithm may work before the encryption after the encryption or during the compression. During the design of encryption algorithm it is major concern that the size of compressed video should not be increased by the encryption. In proposed techniques it is depends on size of buffers, however almost all the statistical properties of video remain unchanged so existing compression techniques works fine. Proposed technique preserves temporal correlation.

\subsection{Codec portability}

It is recommended that an encryption algorithm should work in compliance to video codec and does not require a modification of the underlying video codec/implied framework. Proposed technique works on raw digital video prior to the compression, which makes it codec independent and make fit for all codec.

\subsection{Syntax compliance}

A video stream compressed with a codec contains syntax structure respective to codec, to help the decoder to properly interpret the video stream. It is recommended that an encryption algorithm is able to preserve the syntax structure for the encrypted video stream so that it is decidable at the receiver side without decryption. Before compression algorithms are inherently syntax compliant.

\subsection{Visual degradation}

This is required to measure perceptual distortion of video stream. For sensitive video applications like video conferencing, diplomatic dialogue, military communication or business meetings needs high degree of visual degradation to make it totally incomprehensible for third parties other than communicators while for entertainment applications low visual degradation is needed to keep it comprehensible to user to stimulate for purchase of high quality video.

Proposed technique adds flexibility for service specific security provisioning along with consideration of client device resources.

\subsection{Error tolerance}

A highly desirable feature of encryption algorithm especially critical when data travelled through error prone networks. Some of encryption techniques have strong avalanche effect (error in one encrypted bit results in more erroneous bits during decryption) hence causes distortion and in some cases lost of important information, which is critically intolerable for some of video application like medical imaging. There is strong need to device error tolerant encryption techniques. Critical requirement when service is accessed over wireless media, highly error prone media. Further, in medical imaging system error tolerant encryption techniques are necessary requirement.

Proposed technique works at byte level independently; therefore error in single bit/byte is not propagated to other neighboring bytes, which makes it error tolerant encryption. 


\subsection{Lossless visual quality}

The encryption should produce same visual quality as original video when decrypted legally. This is one of highly desirable feature for entertainment applications and critical requirement for medical imaging systems.

\subsection{Network neutral design}

Proposed techniques works at application layer at both side, this makes it network independent design. However, network dependent implementation based on RRP is discussed by D. Hooda in [5].

\subsection{Experiment results}

We use MATLAB to simulate the above encryption and decryption algorithms. We run programs on the computer with Processor Intel ${ }^{\circledR}$ Core(TM)2 Duo CPU T5670 @ $1.80 \mathrm{GHz}, 1.00 \mathrm{~GB}$ RAM. Our method uses standard image color model RGB and directly manipulate each frame completely at byte level. In experiment, benchmark video sequence 'Foreman' have been used for the analysis as raw digital video in RGB-24 $(176 \times 144)$ base color model. Experiments are carried out to show different perceptual encryption levels, reconstructed video from the encrypted video stream and decryption time taken by the proposed technique doing so. Study of effect of 'Distortion Factor' on visual degradation is also presented. From the result presented It is evident that the degradation of the visual quality is effectively controlled by the 'Distortion Factor'. video data is only partially degraded when Distortion factor value 1,255 which makes it suitable for entertainment applications while as other values of 'Distortion Factor' produce more disguised video therefore good for communication applications where confidentiality is needed. The results of encrypted frames, decryption of respective encrypted frame and time taken by the algorithm at receiver side which is high end server and decryption time is shown for different values of 'Distortion Factor'.

Personalized video applications are sensitive applications and usually have strict security requirements equal to those demanded for text encryption. The encryption algorithms for personalized video services have to withstand not only classical cryptanalytic attacks but also the perceptual attack $[2,3]$ in order to ensure that no visible information related to the sensitive communication is disclosed. Same is achieved by selecting appropriate 'Distortion Factor'

Distortion Factor $=1$
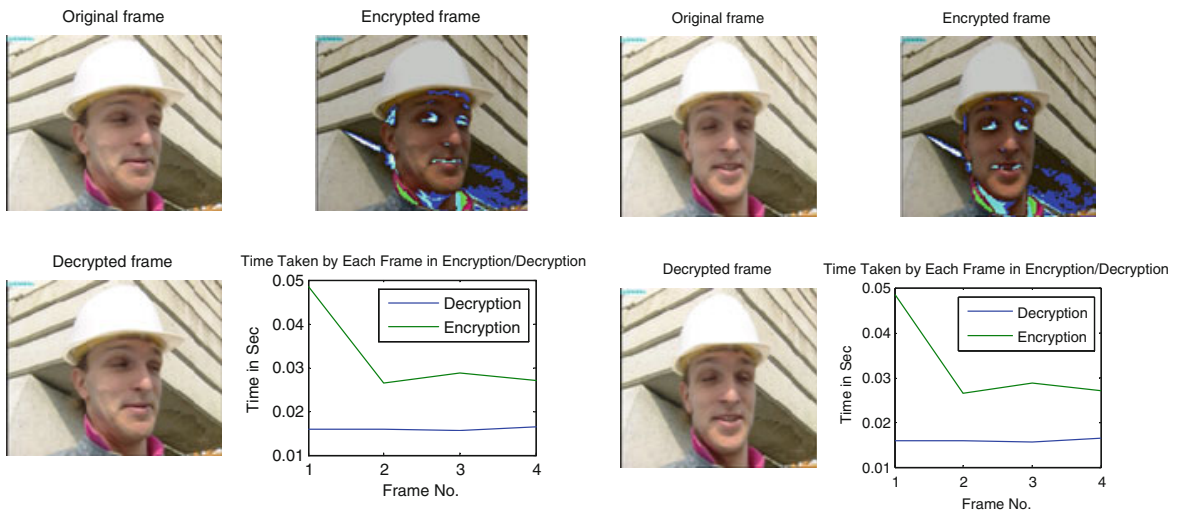

Distortion Factor $=3$
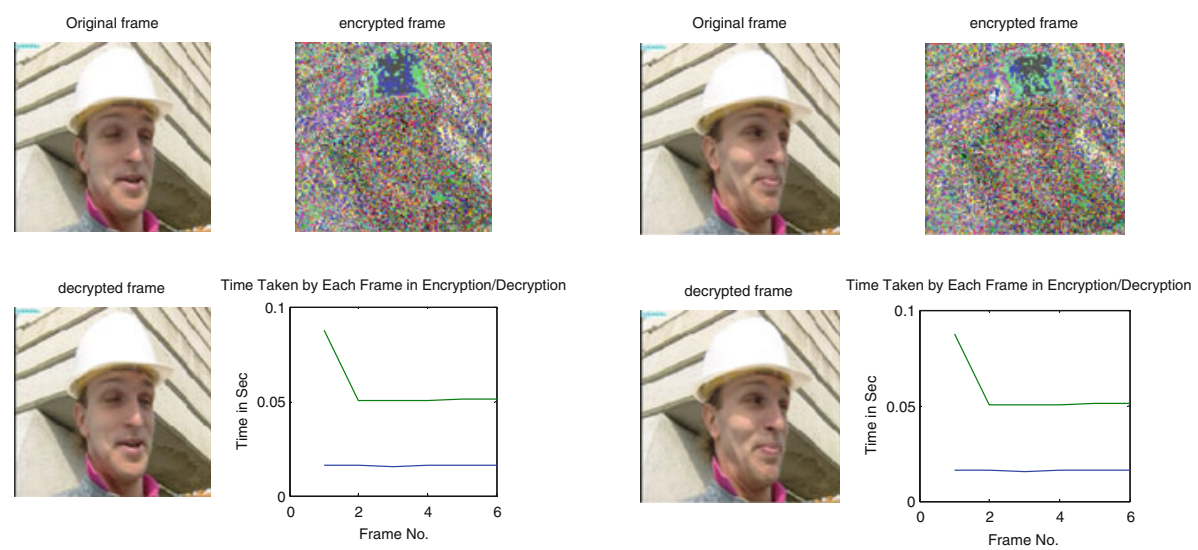
Distortion Factor=7
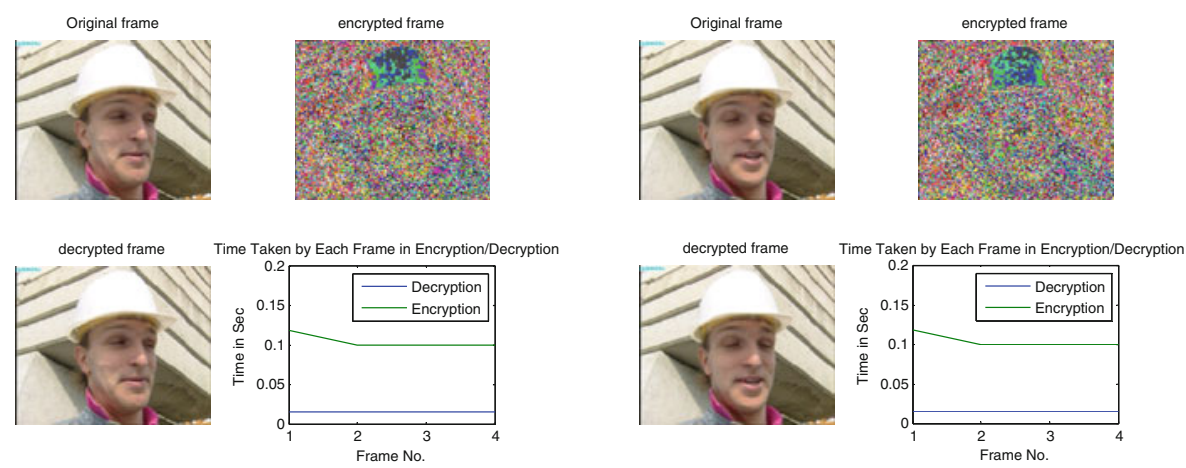

Distortion Factor $=11$
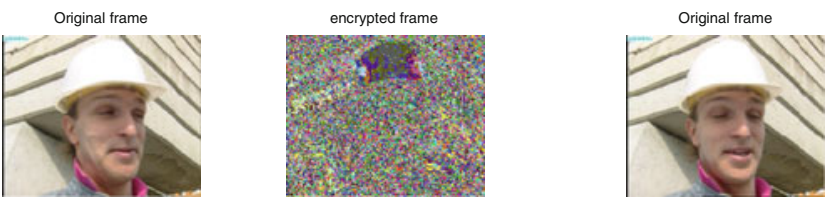

encrypted frame
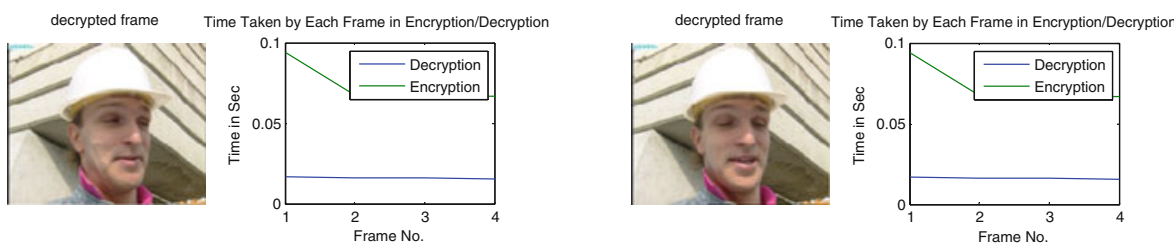

Distortion Factor=19
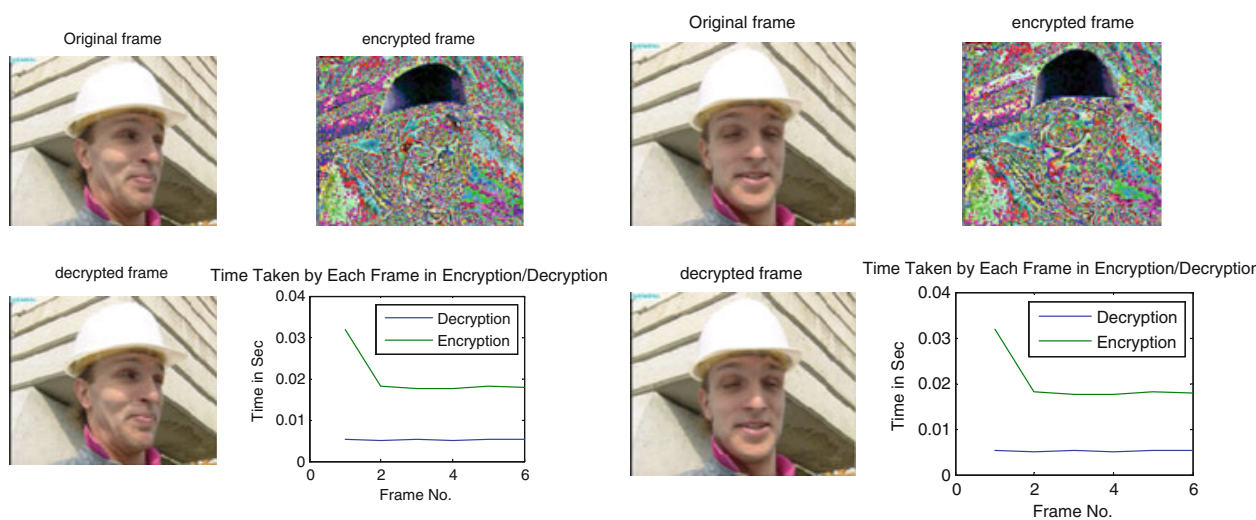

Distortion Factor $=51$
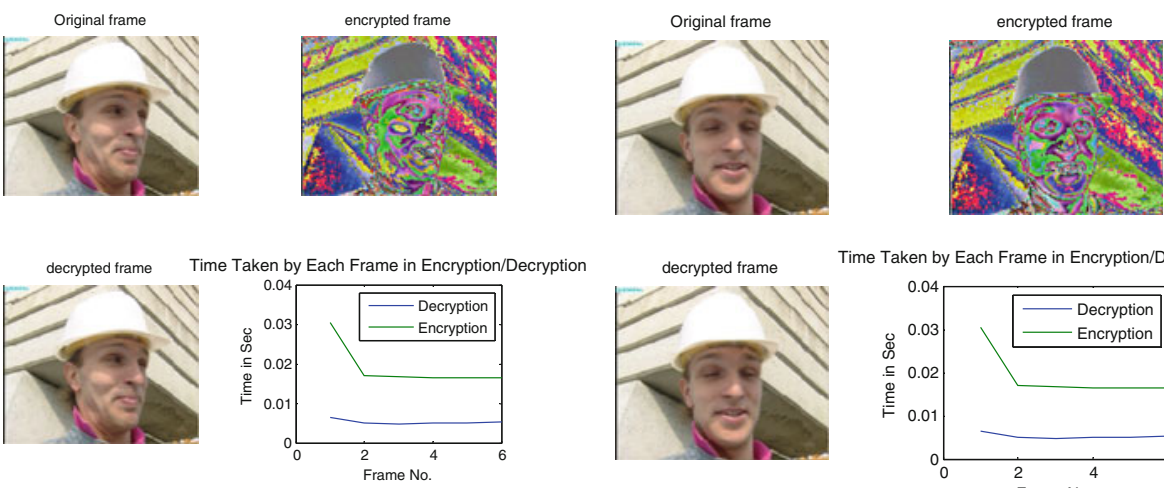

Time Taken by Each Frame in Encryption/Decryption

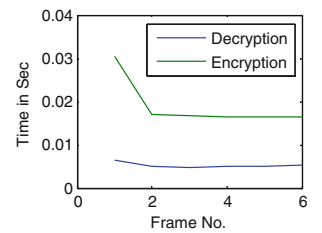




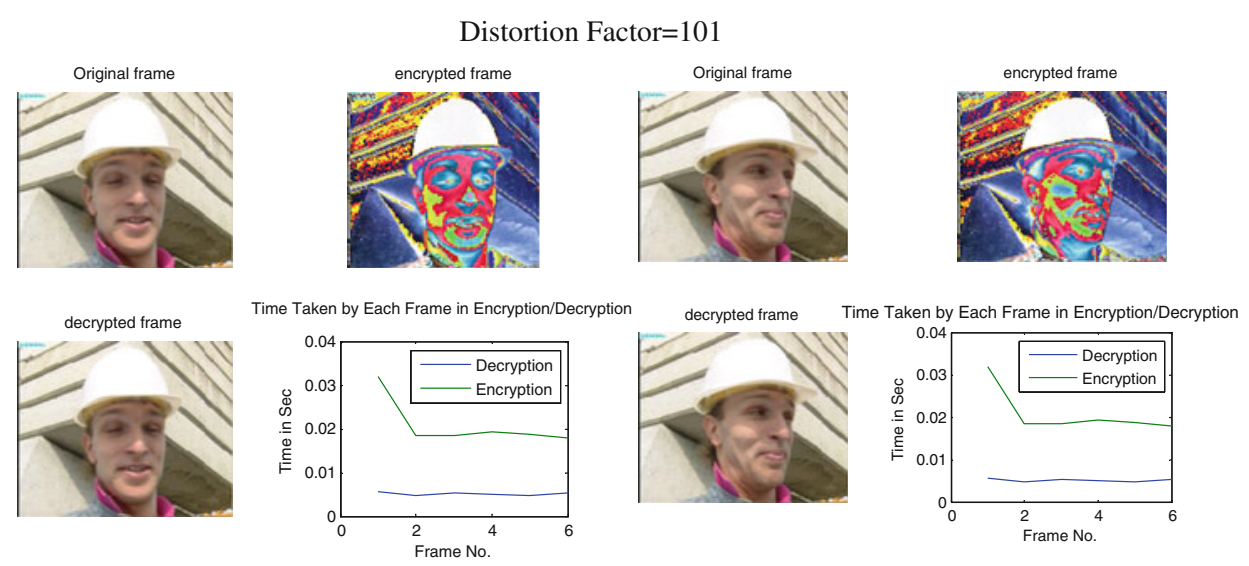

Distortion Factor $=255$
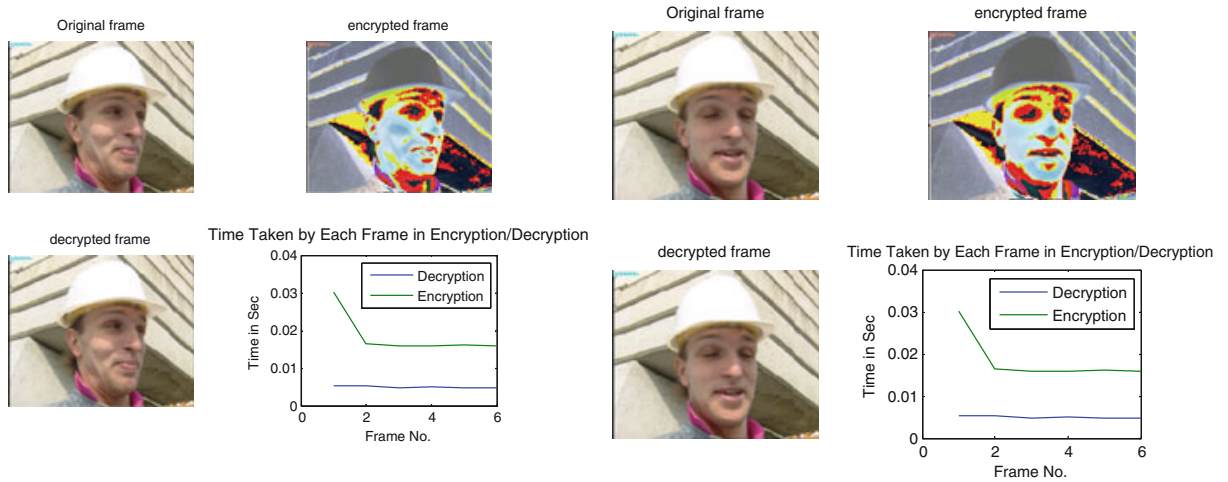

From above figures following is concluded about the proposed parameterized technique:

1 Decryption time for proposed technique is estimated $.005 \mathrm{~s}$ per frame (app.) and constant for each frame. One of the foremost goal of this work to meet security requirements of different multimedia services while used in constrained environment.

2 The degradation of the visual quality is effectively controlled by the 'Distortion Factor'. Video data is only partially degraded when Distortion factor value 1,255 which makes it suitable for entertainment applications while as other values of Distortion factor produce more disguised video therefore good for communication applications where confidentiality is needed. Degradation of visual quality varies with Distortion Factor.

3 'Distortion Factor' with respect to the buffer size contributes effectively for perceptual degradation of video. Here it is established that maximum perceptual degradation is obtained when

\section{$\frac{\text { Buffer Size }}{\text { Distortion Factor }} \approx$ Distortion Factor}

From this it is concluded that when Distortion Factor $=\sqrt{\text { Buffer Size }}$, it generates highly disguised video.
4 Further, more high level of security may be achieved by taking different buffers for each color or alternatively for even and odd pixels. This scheme establishes tradeoff between security level and memory.

\section{Conclusion}

Current software implementations of cryptographic techniques are slower and hardware implementation restricts the flexibility in selection of algorithm as per security needs of applications. We have proposed a method which incorporates flexibility of software implementation and faster execution of hardware implementation. Proposed security technique offer varying level of perceptual encryption which makes it strong contender to offer different security needs of different multimedia services i.e. controlled access for entertainment applications and confidentiality and privacy for sensitive communication services. Proposed architecture is highly suitable for video streaming applications and offers reasonably good security in real time (on the fly). This approach demonstrates very low processing overhead on video sinks therefore make it suitable for the display devices with limited computational capability; further, its codec independent design makes its 
integration possible with any video codec. There is scope to improve encryption time significantly by adding reference table to video source rather than calculating reference for each video byte.

\section{References}

1. Liu F, Koenig H (2010) A survey of video encryption algorithms. Comput Secur 29:3-15
2. Uhl A, Pommer A (2005) Image and video encryption: from digital rights management to secured personal communication, Advances in information security, vol 15. Springer, Boston

3. Wu CP, Kuo CCJ (2005) Design of integrated multimedia compression and encryption systems. IEEE Trans Multimedia 7(5):828-839

4. Chen WWL (1981) Elementary Number Theory, 2003

5. Hooda D, Singh P (2013) A new approach to design programmable secure network interface card. Intern J Comput Appl 62(8):33-36 